\title{
Understanding the Structure of the Subtropical Thermocline
}

\author{
Jeff A. Polton and David P. Marshall \\ Department of Meteorology, University of Reading, Berkshire, United Kingdom
}

(Manuscript received 3 May 2001, in final form 18 November 2002)

\section{ABSTRACT}

\begin{abstract}
Closing a gyre with a western boundary current imposes a constraint on its vertical structure that requires there to be no net vertical flux of potential vorticity through any closed pressure contour in steady state. This constraint resembles the traditional similarity balance for the internal thermocline, with additional terms representing friction in the western boundary current and convection in the mixed layer. The terms in the integral constraint are diagnosed in a planetary geostrophic ocean model and are used to understand the coexistence of ventilated and internal thermoclines within the subtropical gyre.
\end{abstract}

\section{Introduction}

A double thermocline is a generic stratification feature in each of the subtropical ocean basins. For example, in the North Atlantic (Fig. 1), the strongest density variations are found in both an upper thermocline centered at around 100-200 $\mathrm{m}$ and a lower thermocline centered at around 400-1000 m; these two thermoclines are separated by a region of weakly stratified "mode water."

In broad terms, attempts to explain the structure of the subtropical thermocline have fallen into two distinct classes. In the "ventilated" theory (Luyten et al. 1983), horizontal variations in surface density are adiabatically mapped onto a vertical density structure by the threedimensional circulation, subject to the constraint of material conservation of potential vorticity. Alternatively, in the "internal boundary layer" (or "diffusive") theory (Robinson and Stommel 1959; Stommel and Webster 1962; Salmon 1990), the thermocline is viewed as an internal boundary layer that forms at the interface between the downwelling waters of the subtropical gyre and the upwelling waters of the abyss. Observational estimates show the diapycnal mixing rate $\kappa_{v}$ in the ocean to be small, typically $10^{-5} \mathrm{~m}^{2} \mathrm{~s}^{-1}$ (Ledwell et al. 1993; Toole et al. 1994), suggesting that the thermocline may be considered as adiabatic to leading order. However, Salmon (1990) claims that the adiabatic ventilated thermocline theory of Luyten et al. (1983) is inconsistent with the asymptotic limit, $\kappa_{v} \rightarrow 0$. Instead, Salmon argues that in the adiabatic limit, the ocean would reduce

Corresponding author address: Jeff A. Polton, Department of Meteorology, University of Reading, P.O. Box 243, Reading, Berkshire RG6 6BB, United Kingdom.

E-mail: j.a.polton@rdg.ac.uk to two vertical layers separated by an internal boundary layer.

Motivated by the above, Samelson and Vallis (1997) investigated the interaction between the large-scale flow and weak diapycnal diffusion using a planetary geostrophic ocean model. In the limit of small diapycnal diffusion, they obtained two distinct subtropical thermoclines, which are broadly in accord with observations (Fig. 1). In the vicinity of isopycnals outcrops, Ekman pumping and potential vorticity conservation combine to produce a shallow, adiabatic thermocline, essentially described by the ventilated theory of Luyten et al. (1983). At the base of the gyre, at the confluence of abyssal upwelling and Ekman pumping, lies an intrinsically diffusive internal thermocline whose dynamics are essentially described by the internal boundary layer theory of Stommel and Webster (1962) and Salmon (1990). This separation of thermocline regimes into a nearly adiabatic regime overlying a diffusive regime was anticipated by Welander (1971) and also suggested by the diagnostics of Cox and Bryan (1984) and Colin de Verdière (1989).

The existence of two distinct thermoclines in the Samelson and Vallis model does not necessarily imply that the same processes form these features in the model and in the ocean. For example, in the ocean, the mode water may be formed by subduction of low potential vorticity water along the gyre boundaries where there are sharp gradients in mixed layer properties. Likewise, the lower thermocline may be formed by remote subduction of high-potential-vorticity waters at subpolar latitudes (outside the model domain). Nevertheless, two distinct thermoclines are obtained in the Samelson and Vallis model, and in this note we aim to elucidate their underlying dynamical cause.

The numerical calculations of Samelson and Vallis 


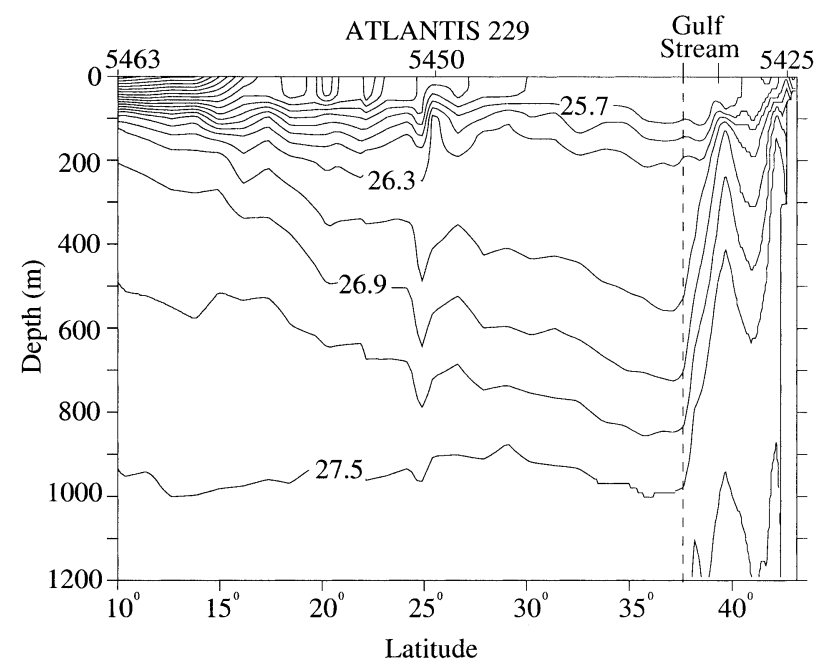

FIG. 1. A meridional cross section of potential density $\left(\sigma_{\theta}\right)$ against depth, in the North Atlantic, showing a double thermocline structure. The section was taken by Atlantis in 1956 along $50^{\circ} \mathrm{W}$; for a full discussion refer to McCartney (1982).

(1997) fundamentally differ from the ventilated and internal boundary layer thermocline theories as they explicitly model the western boundary current. In contrast, both the ventilated and internal boundary layer thermocline theories are open at the western boundary, ensuring that fluid parcels recirculating through the implied western boundary current have lost all memory of their properties on reentering the interior.

Closing a gyre with a western boundary current therefore imposes a strong constraint on its vertical structure. Specifically, Marshall (2000) has shown that, in steady state, the net flux of potential vorticity through any closed Bernoulli potential or density contour at constant depth must vanish:

$$
\iint\left(J_{\text {adv }}+J_{\text {fric }}+J_{\text {buoy }}\right) d A=0,
$$

where $J$ is the vertical flux of potential vorticity that appears in the flux form of the potential vorticity equation (Haynes and McIntyre 1987), with components associated with vertical advection, mechanical forcing, and buoyancy forcing, respectively. The effects of the geostrophic eddies can be included in (1) but are not considered in the present analysis.

If we neglect friction and assume vertical mixing dominates the buoyancy forcing, then to leading order the integral constraint (1) reduces to an integral buoyancy budget between vertical advection and internal mixing:

$$
\iint q^{(z)}\left[w \frac{\partial \rho}{\partial z}-\frac{\partial}{\partial z}\left(k_{v} \frac{\partial \rho}{\partial z}\right)\right] d A \approx 0,
$$

where $q^{(z)}$ is the vertical component of absolute vorticity, $\rho$ is density, and $w$ is the vertical velocity (Marshall
2000). Hence the internal boundary layer thermocline balance, which has hitherto been adopted in an ad hoc manner, has a fundamental theoretical justification in terms of an integral constraint on the structure of a closed gyre although in practice convection, friction, and other dissipative processes can modify this balance.

In this paper, we diagnose the three components of the potential vorticity flux integral constraint (1) in a closed planetary geostrophic ocean. The planetary geostrophic ocean model and integral constraint diagnostic methodology are described in section 2 . The model results and diagnosed potential vorticity fluxes are presented in section 3. A brief concluding discussion is given in section 4 .

\section{Methodology}

\section{a. The planetary geostrophic model}

We use the Samelson and Vallis (1997) model to numerically solve the planetary geostrophic equations in a rectangular hemispheric $\beta$-plane basin. The model is forced by a zonal wind stress that varies with latitude and by a surface heat flux that is proportional to the difference between the sea surface temperature and a reference "equilibrium" temperature.

Within the interior, the equations of motion are

$$
\begin{aligned}
f \mathbf{k} \times \mathbf{v}+\frac{1}{\rho_{0}} \boldsymbol{\nabla}_{h} p & =\mathbf{F}, \\
\frac{\partial p}{\partial z}+\rho g & =0, \\
\frac{\partial \rho}{\partial t}+\mathbf{v} \cdot \nabla_{h} \rho+w \frac{\partial \rho}{\partial z} & =\mathcal{B}, \quad \text { and } \\
\boldsymbol{\nabla}_{h} \cdot \mathbf{v}+\frac{\partial w}{\partial z} & =0 .
\end{aligned}
$$

Friction $\mathbf{F}$ is represented as a linear drag, with coefficient $\eta$ :

$$
\mathbf{F}=-\eta \mathbf{v}
$$

Beneath the surface mixed layer, buoyancy forcing $\mathcal{B}$ is represented by vertical and horizontal diffusion of density, the latter consisting of both Laplacian and biharmonic components:

$$
\mathcal{B}=\kappa_{v} \frac{\partial^{2} \rho}{\partial z^{2}}+\kappa_{h} \nabla_{h}^{2} \rho-\nabla_{h}^{4} \rho,
$$

and with an additional convection scheme that maintains hydrostatic balance through vertical homogenization of the unstable fluid at each time step. Note that no attempt is made to represent the effects of geostrophic eddies in the buoyancy budget, (8). The remaining symbols are defined as follows: $\mathbf{v}$ and $w$ are the horizontal and vertical velocity components, $\rho$ and $\rho_{0}$ are the density and the reference density, $p$ is the pressure, $f$ is the Coriolis 


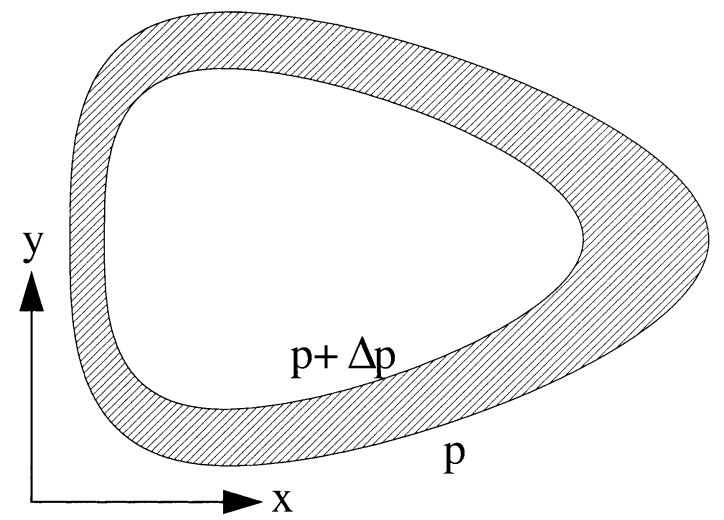

FIG. 2. The average vertical flux of potential vorticity $\bar{J}^{p}$ is calculated over the shaded area between two adjacent pressure contours, $p$ and $p+\Delta p$.

parameter, and $\mathbf{k}$ is the unit vertical vector. The lateral mixing coefficients, $\kappa_{h}$ and $\lambda$, are both chosen to be small enough, relative to the vertical mixing coefficient $\kappa_{v}$, to ensure that vertical diffusion dominates the diapycnal fluxes but also to be large enough that the lateral boundary layers can be resolved. In this paper the values used are $\kappa_{v}=10^{-5} \mathrm{~m}^{2} \mathrm{~s}^{-1}, \kappa_{h}=10 \mathrm{~m}^{2} \mathrm{~s}^{-1}, \lambda=1.25$ $\times 10^{12} \mathrm{~m}^{4} \mathrm{~s}^{-1}$, and $\eta=4.2 \times 10^{-6} \mathrm{~s}^{-1}$. The model is integrated to a steady state in an idealized rectangular domain of $5000 \mathrm{~km} \times 5000 \mathrm{~km} \times 5 \mathrm{~km}$, centered at $35^{\circ} \mathrm{N}$, and discretized on a grid of $65 \times 65 \times 66$ grid points. Further model details, including surface and lateral boundary conditions, are as given in Samelson and Vallis (1997).

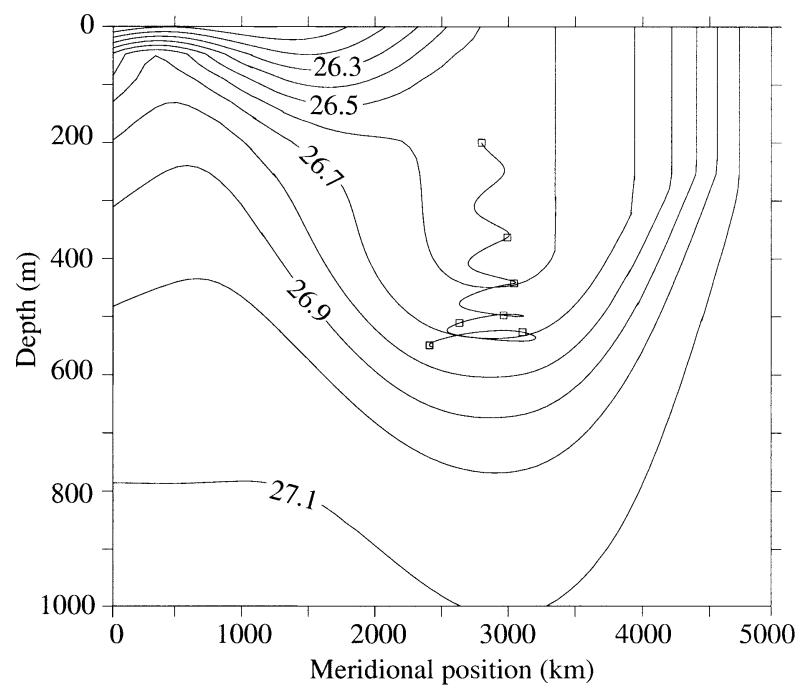

FIG. 3. A meridional cross section $1000 \mathrm{~km}$ from the western boundary shows the potential density in the planetary geostrophic subtropical gyre to have a realistic double thermocline structure. Labeled with $20-y r$ markers, a corkscrew trajectory that recirculates through the subtropical mode water into the internal thermocline is projected onto this cross section.

\section{b. Potential vorticity flux constraint in a planetary geostrophic ocean}

In the planetary geostrophic ocean, a number of simplifications arise in the integral constraint (1). The three components of the potential vorticity flux are given by

$$
\begin{aligned}
J_{\text {adv }} & =\rho_{0} Q w, \\
J_{\text {fric }} & =\mathbf{F} \times \nabla \rho \cdot \mathbf{k}, \quad \text { and } \\
J_{\text {buoy }} & =f \mathcal{B},
\end{aligned}
$$

where

$$
Q=-\frac{f}{\rho_{0}} \frac{\partial \rho}{\partial z}
$$

is the large-scale potential vorticity and the integral is taken over an area bound by a closed pressure (or density) contour at constant depth. The buoyancy-forced, $J_{\text {buoy }}$, component of the potential vorticity flux is further decomposed into two separate components: $J_{\text {conv }}$, associated with convection, and $J_{\text {mix }}$, associated with the other terms as given in (8).

In evaluating (1) at different levels, the integration is performed over the area $A$ between two adjacent pressure contours, $p$ and $p+\Delta p$ (Fig. 2). The integrated fluxes are then normalized by the integration area to define a new quantity:

$$
\bar{J}^{p}=\lim _{\Delta p \rightarrow 0} \frac{\int J d A}{\int d A} .
$$

The latter refinement produces area-weighted averages of the potential vorticity fluxes between adjacent contours, allowing direct comparison between the magnitudes of the fluxes at different depths and pressure values.

\section{Results}

\section{a. Thermocline structure and fluid trajectories}

Before diagnosing the vertical potential vorticity fluxes, we present a meridional cross section of potential density through the subtropical gyre (Fig. 3); this double thermocline structure compares favorably with observational data (Fig. 1). Superimposed on the potential density section is the trajectory (meridional and vertical displacements) of a fluid parcel recirculating through the subtropical mode water and into the internal thermocline. If the flow were purely geostrophic, then the fluid parcel would simply recirculate around closed pressure contours at constant depth. However, as found by Cox and Bryan (1984) and Spall (1992), the fluid parcel follows a corkscrew trajectory, spiralling downward because of Ekman pumping and outward because of the frictional ageostrophic velocity, 


$$
\mathbf{v}_{\mathrm{ag}}=-\frac{\mathbf{k} \times \mathbf{F}}{f} .
$$

Owing to the bowled nature of the isopycnals, both of these ageostrophic displacements advect the fluid parcel from a region of low density to a region of higher density. In steady state, the ageostrophic advection of potential density must therefore be compensated by a net decrease in the buoyancy of the fluid parcel.

An explicit link between ageostrophic advection of potential density and vertical potential vorticity fluxes can be made by rewriting the integral constraint, (1) combined with (9)-(11), in the following form (Marshall 2000):

$$
\iint f\left(\mathbf{v}_{\text {ag }} \cdot \nabla_{h} \rho+w \frac{\partial \rho}{\partial z}-\kappa_{v} \frac{\partial^{2} \rho}{\partial z^{2}}\right) d A \approx 0 .
$$

Here the horizontal mixing terms in (8) have been neglected because they turn out to be small relative to the vertical mixing term in the present model calculation. Note, however, that horizontal mixing across sloping density surfaces can represent the dominant component of diapycnal mixing in many models (Veronis 1975), including those in which geostrophic eddies are resolved (Roberts and Marshall 1998).

Physically, in a subtropical gyre, the first two terms in (15) advect potential density outward and downward. Both act to decrease the potential vorticity near the surface and increase the potential vorticity at depth. ${ }^{1}$ Thus there is a net downward flux of potential vorticity, which must be balanced by a flux of potential vorticity associated with buoyancy forcing.

The crucial ingredient in (15), as compared for example with the earlier diagnostics of Colin de Verdière $(1988,1989)$, is the absence of a geostrophic advection term in (15). Thus, while geostrophic advection may locally dominate the buoyancy budget, in an integral sense, the ageostrophic advection of potential density must balance the net buoyancy forcing (with each term weighted by the Coriolis parameter). This integral buoyancy balance is mathematically equivalent to the integral constraint on the vertical potential vorticity fluxes (Polton 2002) discussed in the following. This ageostrophic buoyancy balance was hinted at by Colin de Verdière (1989) in his decomposition of the horizontal circulation into rotational and divergent components.

\section{b. Vertical potential vorticity fluxes}

To illustrate the different dynamical regimes, we now present analyses at 90, 400, 580, and $700 \mathrm{~m}$, corresponding to the ventilated thermocline, mode water, internal thermocline, and the upper limit of the abyss,

\footnotetext{
${ }^{1}$ The full three-dimensional frictional potential vorticity flux is directed along inclined isopycnals toward the center of the gyre. Since the isopycnals bowl downward, the vertical component of the frictional flux is therefore downward.
}

respectively. At each of these levels $\bar{J}^{p}$ is calculated for all components, taken from the smallest closed pressure contour, at the center of the gyre, to the largest closed pressure contour (Figs. 4ai-di). The $\bar{J}^{p}$ components are then plotted against the fractional area of the domain enclosed by the corresponding integrating pressure contour (Figs. 4aii-dii). In Figs. 5-8, we show the horizontal structure of the individual potential vorticity flux components at each of the integration depths. Note that the numerical closure is good for all the depths, that is, the sum of the $\bar{J}^{p}$ components, labeled "total," is close to zero. This justifies our steady-state assumption, as well as the integrity of the numerical integration.

\section{1) Ventilated Regime}

In the upper $200 \mathrm{~m}$ the dominant integral balance is between the downward flux of potential vorticity due to strong vertical advection, $\bar{J}_{\text {adv }}^{p}$, and an upward flux of potential vorticity due to convective buoyancy loss, $\bar{J}_{\text {conv }}^{p}$, (Fig. 4ai). The flux of potential vorticity due to friction is smaller but becomes significant away from the center of the gyre, where it reinforces the downward advective flux [as anticipated by Marshall (2000)].

The horizontal structure of the potential vorticity flux components at $90 \mathrm{~m}$, shown in Fig. 5, is qualitatively similar to the heat budget analysis of Colin de Verdière (1989; Fig. 6). The advective flux $J_{\text {adv }}$ vanishes within the mixed layer where there is no stratification and is downward within the ventilated thermocline. There is a small region within the western boundary current where the advective flux is upward, but this is not significant in terms of the integral constraint. The frictional flux $J_{\text {fric }}$ is largest within the western boundary current and is mainly directed downward. These downward advective and frictional fluxes are balanced in the integral constraint (1) by an upward convective flux $J_{\text {conv }}$ within the mixed layer. This is in accord with ventilated thermocline theory of Luyten et al. (1983), in which warm fluid is pumped adiabatically down into the gyre interior and buoyancy forcing is confined to the mixed layer.

It is of interest to compare these results with threedimensional potential vorticity flux budgets of Marshall and Nurser (1992). Within the mixed layer, in regions of subduction, they found a downward convective potential vorticity flux. Beneath the mixed layer, they found an advective potential vorticity flux with a downward component. The latter is consistent with our results. However the convective potential vorticity flux within the mixed layer is upward in our diagnostics. The reasons for this difference are as follows: 1) Marshall and Nurser did not close their gyre with a western boundary current, and hence their model does not need to satisfy the integral constraint (1). 2) Marshall and Nurser did not model regions in which fluid is entrained into the mixed layer, where the convective potential vorticity flux is directed upward. 3) In regions of surface 
ai) $\bar{J}^{p}$ at $90 \mathrm{~m}$

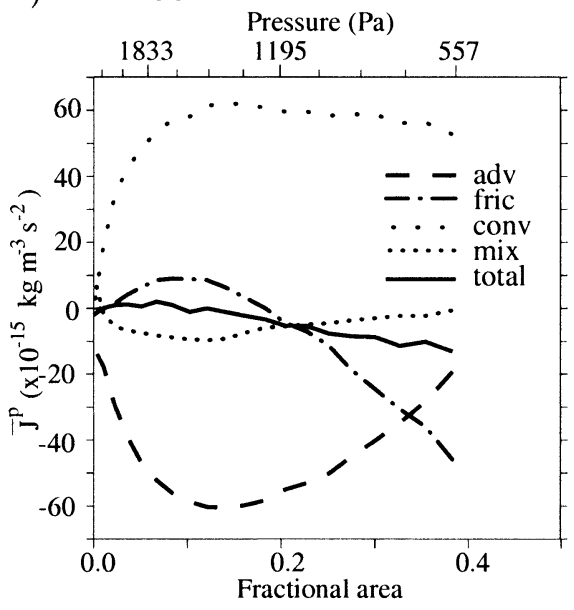

bi) $\bar{J}^{p}$ at $400 \mathrm{~m}$

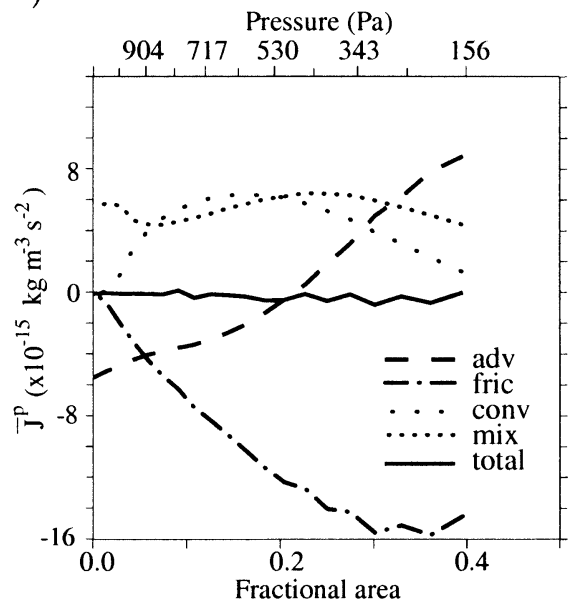

ci) $\bar{J}^{p}$ at $580 \mathrm{~m}$

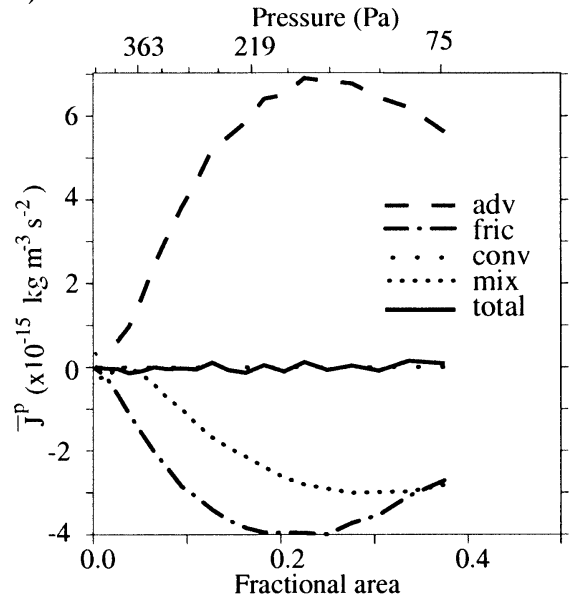

aii)

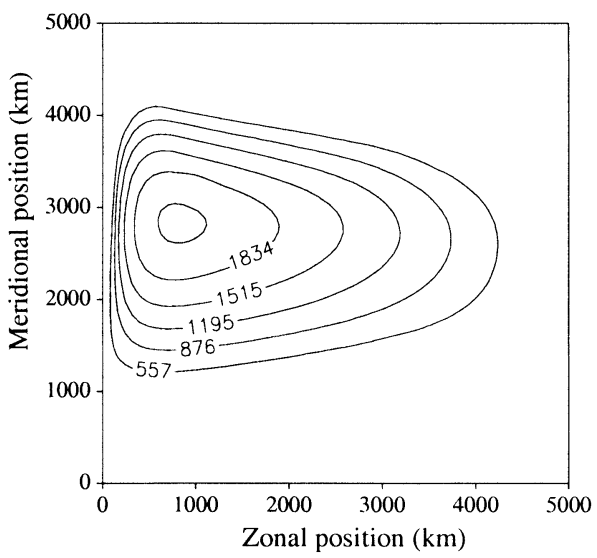

bii)

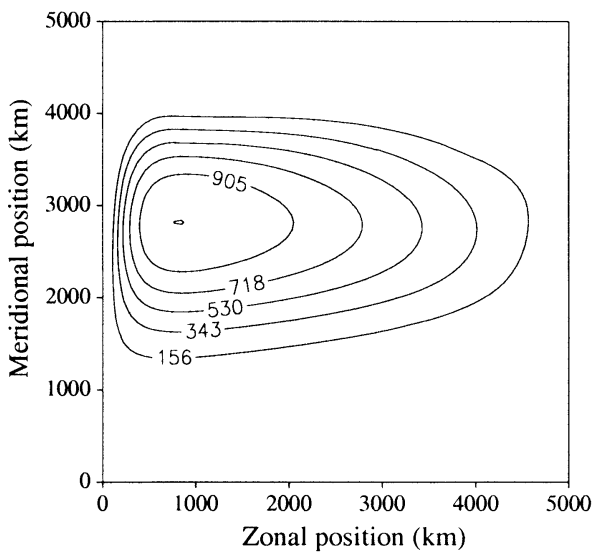

cii)

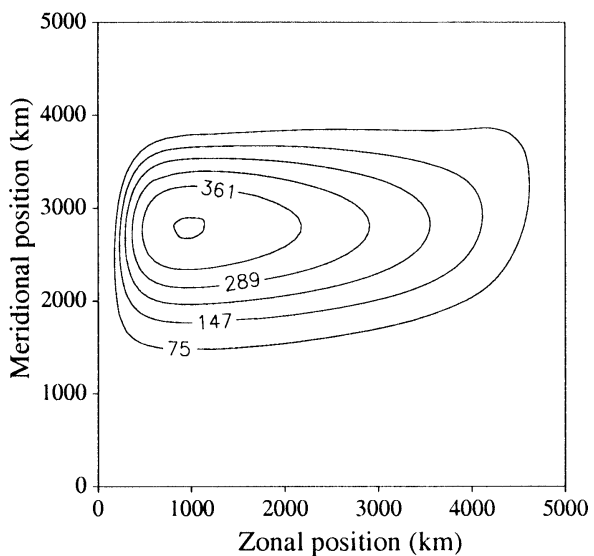

FIG. 4. On the left, the $\bar{J}^{p}$ components are plotted against the fractional area of the domain enclosed by the integration pressure contour (on the lower $x$ axis) and also against the value of the integration pressure contour (on the upper $x$ axis). On the right the range of closed pressure contours, at each depth, from the longest to the shortest, is shown. Pressure $(\mathrm{Pa})$ is given relative to the value at the eastern boundary. The analyses are conducted at depths of (a) 90, (b) 400, (c) 580, and (d) $700 \mathrm{~m}$. These depths correspond to the ventilated thermocline, mode water, internal thermocline, and abyss, respectively (see Fig. 3). 
di) $\bar{J}^{p}$ at $700 \mathrm{~m}$

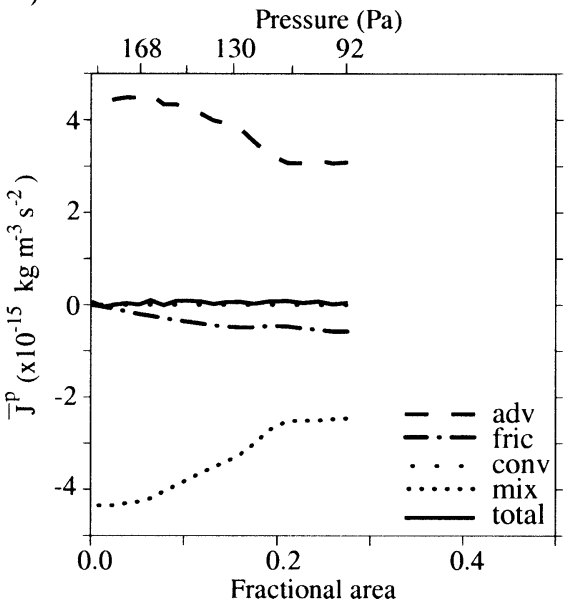

dii)

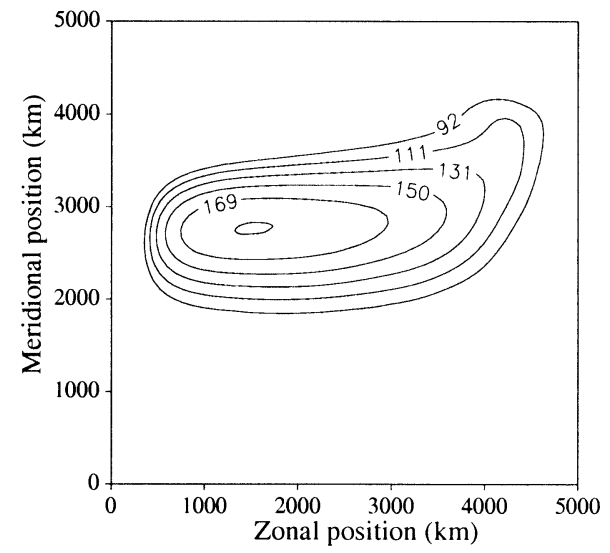

FIG. 4. (Continued)

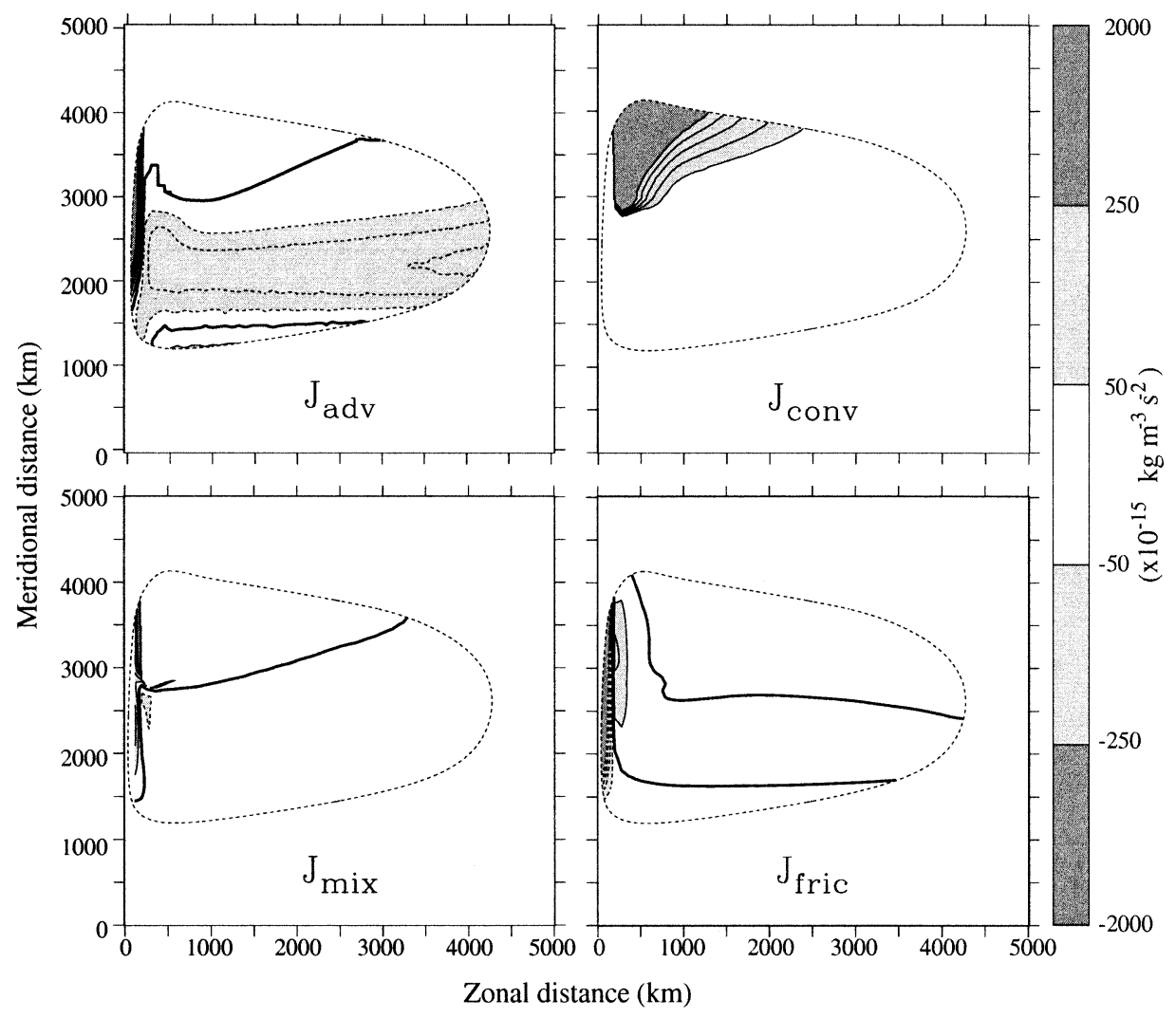

FIG. 5. The ventilated thermocline. The vertical components of the potential vorticity flux vector are shown at a depth of $90 \mathrm{~m}$. Contours are shown for values with a modulus less than $250 \times 10^{-15} \mathrm{~kg} \mathrm{~m}^{-3}$ $\mathrm{s}^{-2}$ with a contour interval of $50 \times 10^{-15} \mathrm{~kg} \mathrm{~m}^{-3} \mathrm{~s}^{-2}$. Dashed contours denote negative values. A heavy black contour denotes zero. The figure is shaded in two tones, with an intensity corresponding to the magnitude of the modulus-light gray: $50-250 \times 10^{-15} \mathrm{~kg} \mathrm{~m}^{-3} \mathrm{~s}^{-2}$ and dark gray: $>250 \times 10^{-15} \mathrm{~kg}$ $\mathrm{m}^{-3} \mathrm{~s}^{-2}$. The dominant balance is between a negative advective term and a positive convection term. Friction is also significant toward the edge of the gyre. 


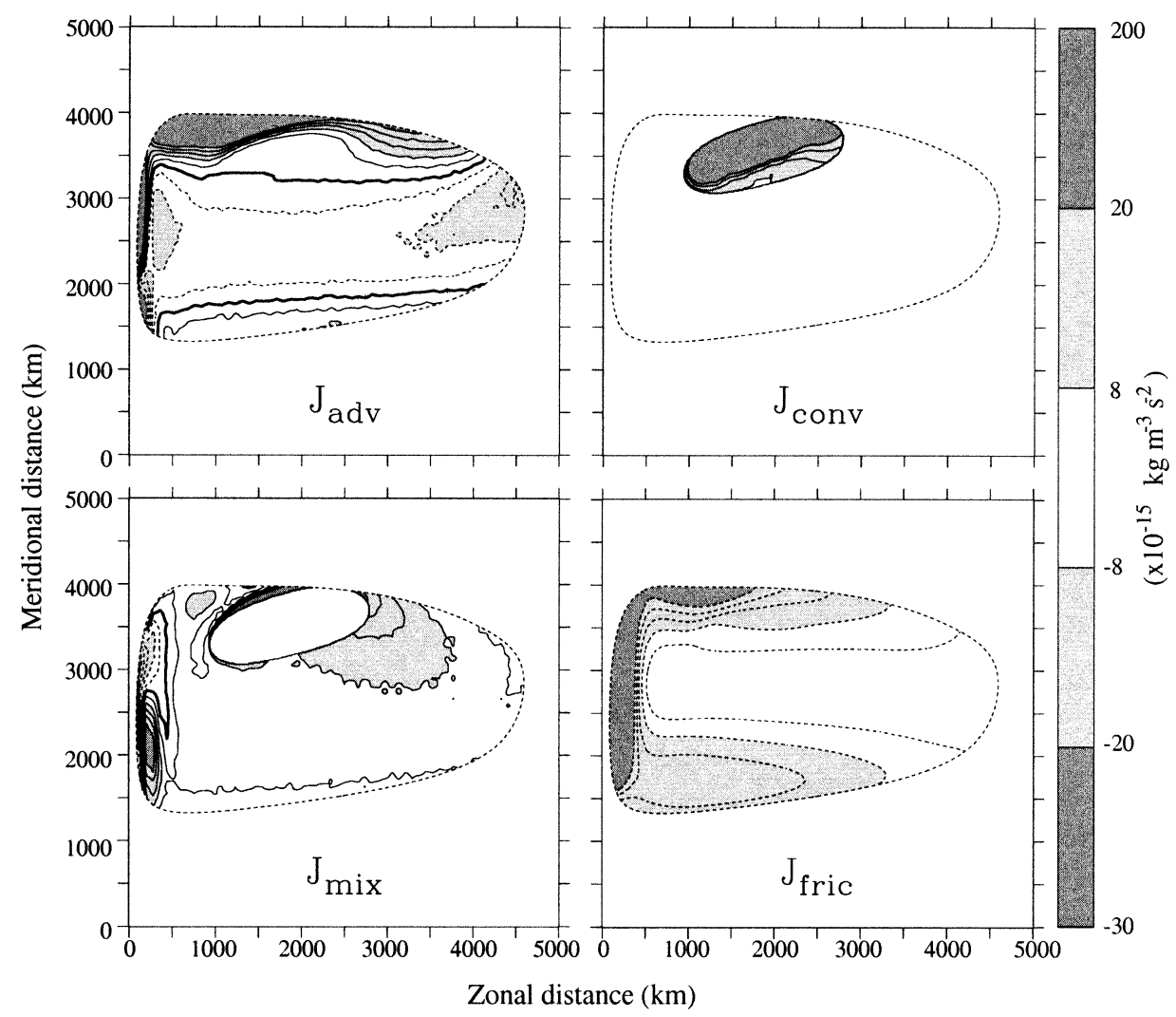

FIG. 6. The mode water. The vertical components of the potential vorticity flux vector are shown at a depth of $400 \mathrm{~m}$. Contours are shown for values with a modulus less than $20 \times 10^{-15} \mathrm{~kg} \mathrm{~m}^{-3} \mathrm{~s}^{-2}$ with a contour interval of $4 \times 10^{-15} \mathrm{~kg} \mathrm{~m}^{-3} \mathrm{~s}^{-2}$. Dashed contours denote negative values. A heavy black contour denotes zero. The figure is shaded in two tones, with an intensity corresponding to the magnitude of the modulus-light gray: 8-20 $\left(\times 10^{-15} \mathrm{~kg} \mathrm{~m}^{-3} \mathrm{~s}^{-2}\right)$ and dark gray: $>20 \times 10^{-15} \mathrm{~kg} \mathrm{~m}^{-3} \mathrm{~s}^{-2}$. Convection now occurs in a restricted area in the northern part of the gyre. The dominant balance must include friction, the only negative flux.

heating, this heat is distributed within only the upper grid cell in the Samelson and Vallis model, whereas this heat is distributed (somewhat artificially) throughout the entire depth of the mixed layer in the Marshall and Nurser model. In the present calculations, convection is therefore confined to regions in which the mixed layer is cooled, and the convective potential vorticity flux is always upward.

\section{2) Mode water}

Analysis at the depth of around $400 \mathrm{~m}$ includes both the recirculating and ventilated mode waters identified by Samelson and Vallis (1997). There is a complicated four-way balance in the integral potential vorticity flux constraint (Fig. 4bi). Note that each term is an order of magnitude smaller than in the ventilated regime.

Near the center of the gyre, corresponding to the recirculating mode water, the convective flux is negligible because the closed pressure contours do not intersect the mixed layer. The balance is between the downward advective flux, $\bar{J}_{\text {adv }}^{p}$, and an upward flux due to mixing, $\bar{J}_{\text {mix }}^{p}$. The center of the gyre is a stagnation point, en- suring that the frictional force (represented as a simple linear drag) vanishes. Thus, at the center of the gyre the friction term vanishes and the integral constraint reduces to precisely the same balance as assumed in the internal boundary layer thermocline theories; that is (1) reduces locally to

$$
w \frac{\partial \rho}{\partial z}-\kappa_{v} \frac{\partial^{2} \rho}{\partial z^{2}}=0
$$

(strictly this balance should include a lateral diffusion term, but this has been chosen to be small in this model). Since $w$ is large and $\kappa_{v}$ is small at the depth of the recirculating mode water, (16) can only be satisfied if the stratification, $\partial \rho / \partial z$, is also small; this, in turn, implies that a mode water must exist.

Farther away from the center of the gyre, corresponding to the ventilated mode water, $\bar{J}_{\text {conv }}^{p}$ becomes significant as the pressure contours intersect the mixed layer (Fig. 6). The advective flux $\bar{J}_{\text {adv }}^{p}$ changes sign because of upwelling on the northern flank of the gyre; however, this is more than compensated for by a downward frictional flux $\bar{J}_{\text {fric }}^{p}$. In terms of the alternative form of the 


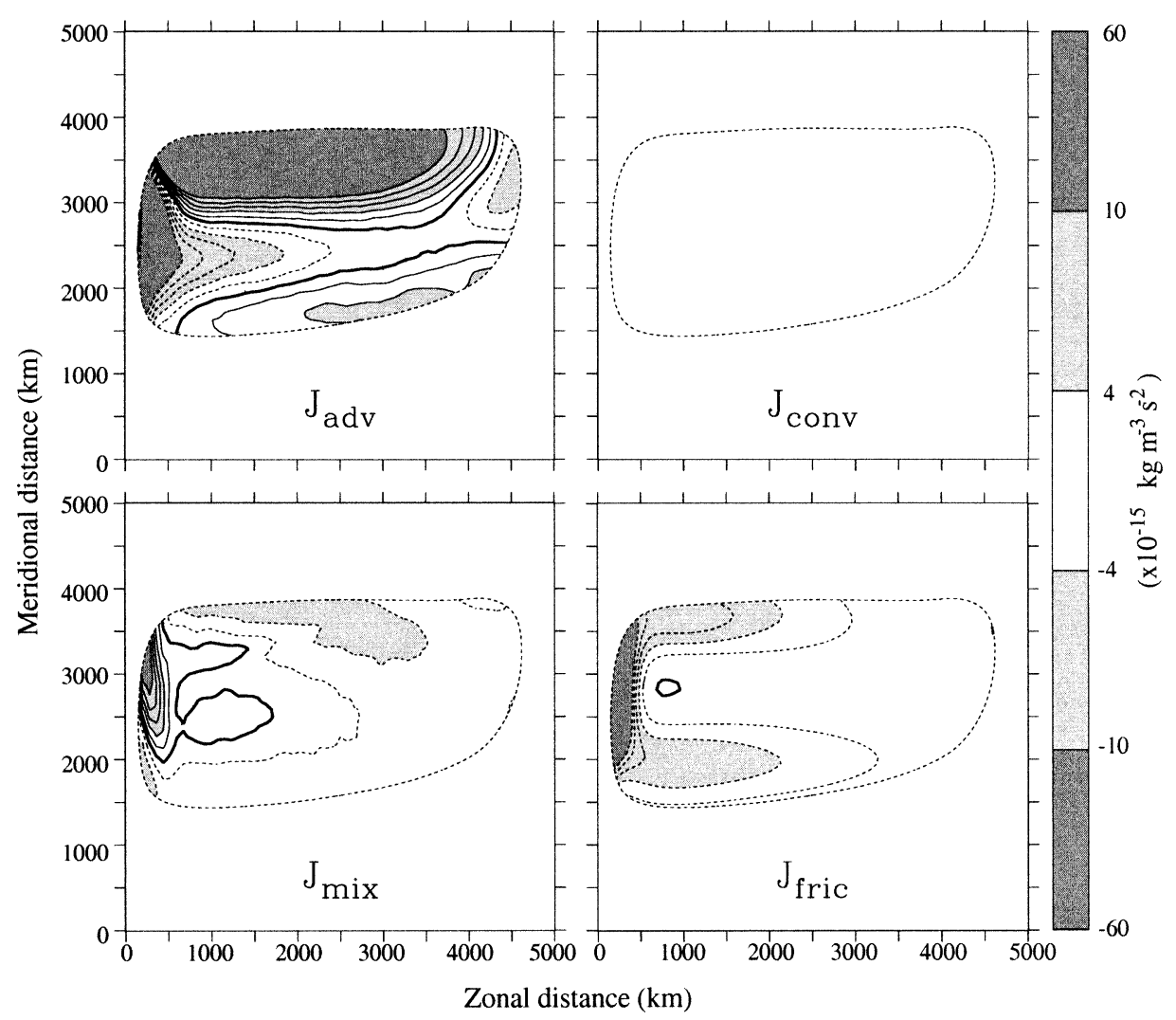

FIG. 7. The internal thermocline. The vertical components of the potential vorticity flux vector are shown at a depth of $580 \mathrm{~m}$. Contours are shown for values with a modulus less than $10 \times 10^{-15} \mathrm{~kg} \mathrm{~m}^{-3}$ $\mathrm{s}^{-2}$ with a contour interval of $2 \times 10^{-15} \mathrm{~kg} \mathrm{~m}^{-3} \mathrm{~s}^{-2}$. Dashed contours denote negative values. A heavy black contour denotes zero. The figure is shaded in two tones, with an intensity corresponding to the magnitude of the modulus-light gray: $4-10\left(\times 10^{-15} \mathrm{~kg} \mathrm{~m}^{-3} \mathrm{~s}^{-2}\right)$ and dark gray: $>10 \times 10^{-15} \mathrm{~kg} \mathrm{~m}^{-3}$ $\mathrm{s}^{-2}$. There is no convection at this depth. The dominant balance, as shown by the shading, is three way and between $J_{\text {adv }}, J_{\text {mix }}$, and $J_{\text {fric }}$. Some smoothing has been applied to the fields to aid visualization.

integral constraint (15), the dominant balance is between lateral ageostrophic advection across sloping isopycnals and mixing. This differs from the classical internal boundary layer thermocline balance (16), which neglects the contribution of friction.

\section{3) INTERNAL THERMOCLINE}

Below the influence of deep convection, the potential vorticity flux integral constraint can only be between $\bar{J}_{\text {adv }}^{p} \bar{J}_{\text {mix }}^{p}$, and $\bar{J}_{\text {fric }}^{p}$. Here we analyze the potential vorticity fluxes at a depth of $580 \mathrm{~m}$. At this depth, in the center of the gyre, the vertical velocity vanishes and hence an internal boundary layer exists where $\bar{J}_{\text {adv }}^{p}$ vanishes. Furthermore, since $\bar{J}_{\text {fric }}^{p}$ is zero at the center of the gyre, this implies that $\bar{J}_{\text {mix }}^{p}$ must also be zero. This is consistent with the internal boundary layer thermocline theory picture (16) whereby the thermocline, defined as the region of most intense stratification $\left(\partial^{2} \rho / \partial z^{2}=0\right)$, is at the confluence of the downwelling and upwelling flows.

In isopycnic coordinates, moving away from the center of the gyre is akin to moving upward. This is due to the downwardly bowled nature of the isopycnals (Fig. 3 ) and the flow being isopycnic to first-order approximation. Similarly, moving away from the center of the gyre at a fixed depth is akin to moving downward in the gyre, and moving into denser water. This explains the increase in the $\bar{J}_{\text {adv }}^{p}$ toward the edge of the gyre (Fig. 7 ), as the strength of the abyssal upwelling increases from zero. Also, farther away from the center of the gyre, $\bar{J}_{\text {fric }}^{p}$ becomes significant in the integral constraint and so only when $\bar{J}_{\text {fric }}^{p}$ and $\bar{J}_{\text {mix }}^{p}$ are combined is the strong upward $\bar{J}_{\text {adv }}^{p}$ flux balanced (Fig. 4ci). As in the mode water, this differs from the classical thermocline balance (16), which neglects the contribution of friction.

\section{4) AByss}

Last, we show the potential vorticity fluxes at $700 \mathrm{~m}$, which represents the upper limit of the abyssal ocean. The potential vorticity flux integral balance is between a basin-scale upward $\bar{J}_{\text {adv }}^{p}$ and a basin-scale downward $\bar{J}_{\text {mix }}^{p}$. In terms of (15), this is reminiscent of the classical Stommel and Arons (1960) picture in which upwelling 


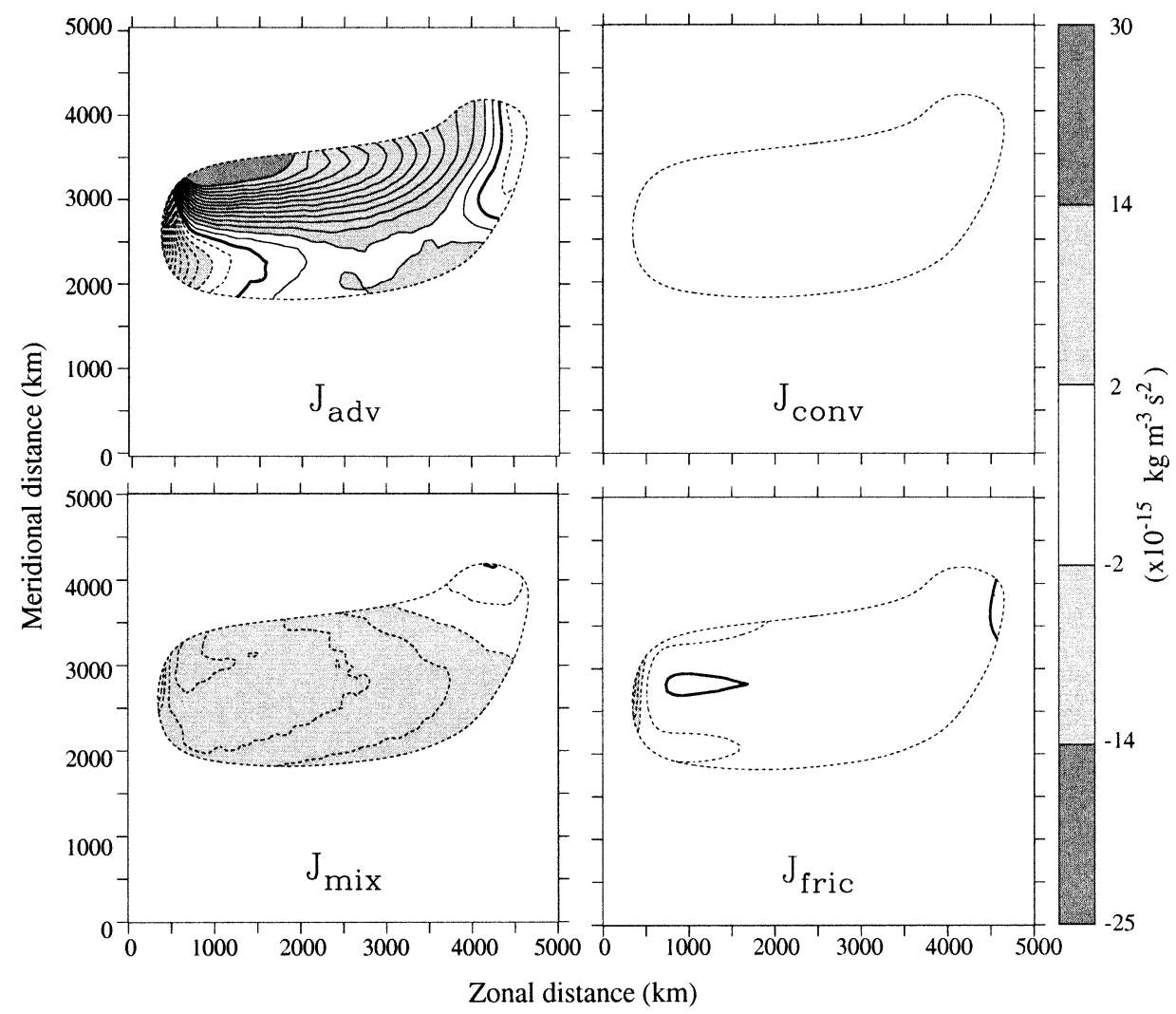

FIG. 8. The upper limit of the abyss. The vertical components of the potential vorticity flux vector are shown at a depth of $700 \mathrm{~m}$. Contours are shown for values with a modulus less than $14 \times 10^{-15} \mathrm{~kg} \mathrm{~m}^{-3}$ $\mathrm{s}^{-2}$ with a contour interval of $10^{-15} \mathrm{~kg} \mathrm{~m}^{-3} \mathrm{~s}^{-2}$. Dashed contours denote negative values. A heavy black contour denotes zero. The figure is shaded in two tones, with an intensity corresponding to the magnitude of the modulus-light gray: 2-14 $\left(\times 10^{-15} \mathrm{~kg} \mathrm{~m}^{-3} \mathrm{~s}^{-2}\right)$ and dark gray: $>14 \times 10^{-15} \mathrm{~kg} \mathrm{~m}^{-3} \mathrm{~s}^{-2}$. There is no convection at this depth. The dominant balance is between a positive advective term and a negative mixing term. Friction is not significant in this balance. Some smoothing has been applied to the fields to aid visualization.

of the cold abyssal waters is balanced by a downward transfer of heat through diapycnal mixing and is also in qualitative agreement with the abyssal heat balance of Colin de Verdière (1989, Fig. 9). The frictional potential vorticity flux $\bar{J}_{\text {fric }}^{p}$ is not found to be significant at these greater depths, partly because the closed pressure contours no longer extend to within $500 \mathrm{~km}$ of the western boundary, where the flow is enhanced and, hence, the friction is greater (cf. Fig. 7 and Fig. 8). However, in the ocean, the abyssal circulation is dominated by boundary currents, and therefore we anticipate that the frictional potential vorticity flux may not generally be negligible.

\section{c. Sensitivity to model coefficients}

The sensitivity of the vertical profile to wind forcing and vertical diffusivity has already been studied in some detail by Samelson and Vallis (1997). We have investigated the dependence of the integral potential vorticity flux budgets to changes in the frictional coefficient and find that the different characteristic regimes, described in this section, are not sensitive to the friction. By contrast, the depths at which these regimes are found are observed to depend on the value of the frictional coefficient.

\section{Concluding remarks}

Closing a gyre with a western boundary current imposes a strong constraint on its vertical structure. This constraint, in the form of an integral balance of potential vorticity fluxes, amalgamates processes spread geographically over the gyre (Ekman pumping over the whole of the gyre, convection in the mixed layer, mixing in the internal thermocline, and friction in the western boundary current). This offers a conceptual advantage over the more traditional pointwise balance by allowing a gyre-scale approach for understanding the ocean's structure. In this paper we have argued that the internal thermocline balance, which has hitherto been adopted locally in an ad hoc manner, emerges as an integral constraint on the structure of the subtropical gyre, even though the dominant local balance is very much in ac- 
cord with the Luyten et al. (1983) ventilated thermocline theory. We have illustrated the different regimes by diagnosing the individual terms of the integral constraint in a planetary geostrophic ocean model. Within the ventilated thermocline, the potential vorticity flux associated with buoyancy forcing in the mixed layer breaks the classical internal boundary layer balance. However, beneath the influence of convection, we have shown that the internal boundary layer balance reemerges, albeit with an additional term involving friction.

A key consequence of the integral constraint is that western boundary currents may play an active role in setting the internal stratification of a gyre. This is important since previous thermocline studies have not included a western boundary current, assuming it only to play a passive role in closing the gyre circulation. The greatest uncertainty in applying our results to the ocean concerns the nature of frictional processes in the western boundary current. In reality, friction represents the action of small-scale turbulence and geostrophic eddies; indeed a recent, alternative, theory to the ventilated and internal thermocline theories (Marshall et al. 2002) proposes eddy-transfer processes as a possible mechanism for setting the ocean stratification. The detailed impact of dissipative processes and eddies on the vertical structure requires further study.

Acknowledgments. Financial support was provided by the National Environment Research Council, GT04/98/ MS/305. We also thank Geoff Vallis and Roger Samelson for making their planetary geostrophic model available. We are also grateful to Ric Williams, Lynne Talley, and the anonymous reviewers for their constructive comments that led to a clearer manuscript.

\section{REFERENCES}

Colin de Verdière, A., 1988: Buoyancy driven planetary flows. $J$. Mar. Res., 46, 215-265.
- 1989: On the interaction of wind and buoyancy driven gyres. J. Mar. Res., 47, 595-633.

Cox, M. D., and K. Bryan, 1984: A numerical model of the ventilated thermocline. J. Phys. Oceanogr., 14, 674-687.

Haynes, P. H., and M. E. McIntyre, 1987: On the evolution of vorticity and potential vorticity in the presence of diabatic heating and frictional or other forces. J. Atmos. Sci., 44, 828-841.

Ledwell, J. R., A. J. Watson, and C. S. Law, 1993: Evidence for slow mixing across the pycnocline from an open-ocean tracer-release experiment. Nature, 364, 701-703.

Luyten, J. R., J. Pedlosky, and H. Stommel, 1983: The ventilated thermocline. J. Phys. Oceanogr., 13, 292-309.

Marshall, D. P., 2000: Vertical fluxes of potential vorticity and the structure of the thermocline. J. Phys. Oceanogr., 30, 3102-3112.

Marshall, J. C., and A. J. G. Nurser, 1992: Fluid dynamics of oceanic thermocline ventilation. J. Phys. Oceanogr., 22, 583-595.

—, H. Jones, R. Karsten, and R. Wardle, 2002: Can eddies set ocean stratification? J. Phys. Oceanogr., 32, 26-38.

McCartney, M. S., 1982: The subtropical recirculation of Mode Waters. J. Mar. Res., 40 (Suppl.), 427-464.

Polton, J. A., 2002: Understanding the vertical structure of the subtropical thermocline. Ph.D. thesis, Department of Meteorology, University of Reading, $129 \mathrm{pp}$.

Roberts, M., and D. Marshall, 1998: Do we require adiabatic dissipation schemes in eddy-resolving ocean models? J. Phys. Oceanogr., 28, 2050-2063.

Robinson, A., and H. Stommel, 1959: The oceanic thermocline and the associated thermohaline circulation. Tellus, 11, 295-308.

Salmon, R., 1990: The thermocline as an "internal boundary layer." J. Mar. Res., 48, 437-469.

Samelson, R. M., and G. K. Vallis, 1997: Large-scale circulation with small diapycnal diffusion: The two-thermocline limit. J. Mar. Res., 55, 223-275.

Spall, M. A., 1992: Cooling spirals and recirculation in the subtropical gyre. J. Phys. Oceanogr., 22, 564-571.

Stommel, H., and A. B. Arons, 1960: On the abyssal circulation of the world ocean. Part II: An idealized model of the circulation pattern and amplitude in oceanic basins. Deep-Sea Res., 6, 217 233.

__ and J. Webster, 1962: Some properties of thermocline equations in a subtropical gyre. J. Mar. Res., 20, 42-56.

Toole, J. M., K. L. Polzin, and R. W. Schmitt, 1994: Estimates of diapycnal mixing in the abyssal ocean. Science, 264, 1120-1123.

Veronis, G., 1975: The role of models in tracer studies. Numerical Models of the Ocean Circulation, National Academy of Science, 133-146.

Welander, P., 1971: The thermocline problem. Philos. Trans. Roy. Soc. London, 270A, 415-421. 